\title{
Improving Aesthetic Outcome in Managing Acute and Chronic Upper and Midface Deformities Using Computer Assisted Planning
}

\author{
MOHAMED A.A. SALEH, M.D., M.R.C.S. (Eng.)*; AMR A. GHANEM, M.D.**; \\ OMAR A. HAMAD, M.D.** and ASSEM M. ABDEL LATIF, M.D., Ph.D.*** \\ The Department of Plastic, Reconstruction, Maxillofacial Surgeries \& Burn Management, Faculty of Medicine*, \\ Ain Shams University, Oral and Maxillofacial Surgery Department, Faculty of Dentistry** and Neurosurgery Department, \\ Faculty of Medicine***, Ain Shams University, Cairo, Egypt
}

\begin{abstract}
Surgical procedures in the facial region are associated by a variety of difficulties. The anatomy of the maxillofacial region, the complexity of the bony architecture as well as the esthetic implications.

The three dimensional imaging and 3D printing, have been applied for the restoration the complex anatomy of craniofacial structures. In addition, mirror-imaging techniques advocating 3D computed tomographic (CT) scanning and 3D printing can maximize the surgical outcome on both the functional and esthetic reconstruction levels. A synthetic scaffold can be pre-molded to the individual prototype skull model to resemble the anatomic contour before applying it to cover the orbital defects.

Our aim is to show the importance and present our experience with three-dimensional virtual planning in solving a variety of acute and chronic clinical deformities within the scope of trauma in the cranio maxillofacial region.
\end{abstract}

25 patients were retrospectively recruited. Mean age was 33.5 years (range 13-59), male: female ratio $=2.6: 1$ (18:7). Eleven patients had acute injuries $(44 \%)$ while the remaining 14 patients $(56 \%)$ had chronic (malunited) fractures performed in 2 different centers treating maxillofacial deformities operated utilizing 3D planning protocols.

The advocation of the virtual planning techniques, three dimension printing and printed custom implant enabled an accurate reduction and fixation procedure of complex acute and chronic complex upper and midface fracture, which is reflected in very satisfactory aesthetic outcome.

Key Words: Three dimension virtual planning - Craniomaxillofacial deformities - Three dimension printing - Three dimension printed custom implant.

\section{INTRODUCTION}

Surgical procedures in the facial region are associated by a variety of difficulties. The anatomy of the maxillofacial region, the complexity of the bony architecture as well as the esthetic implications are the reason for such difficulties. Knowledge in these fields has improved technicalities in surgical procedures of facial region and have imparted their effect on all aspects of treatment [1]. The complexity in surgical execution has even extended to the educational aspects of the practitioner dealing with deformities in this region [2].

In 1980 the advent of three dimensional (3D) computer technologies presented an additional improvement that was set to change the course of surgical treatment in the maxillofacial region forever. The applications of this technology went through several stages of development $[3,4]$. This started with the improvement of the imaging systems and their associated software processing which greatly facilitated visualizing the relevant anatomy preoperatively. This imparted a better description and evaluation of the underlying deformities for the operating team [5].

Stereo lithographic models printed from the modulated images were the next advancement. These were advocated for a variety of clinical applications and included a real time three dimensional realization of the underlying deformity $[3,4]$. Moreover, preoperative hardware adaptation and adjustment was facilitated. This application had its significant advance in operative time, accuracy of plate application and subsequently improved patient outcome [6].

Despite this advantageous advancement in computer application and its inclusion into hospital protocols widely, some criticism was still in order. 
These techniques had room for surgical error and hence this was the demerits that had to be addressed [2].

The next advance included the application of virtual planning. Virtual planning offered the advantage of minimizing human error. Combining virtual planning with the advanced digital printing soft wares gave rise to patient specific implants [3]. The solutions offered by patient specific implants suggest that these techniques will prove beneficial and indispensable to surgeons operating in the maxillofacial region [4].

Recently, 3D imaging and 3D printing, have been applied for the restoration the complex anatomy of craniofacial structures [4]. In addition, mirror-imaging techniques advocating 3D computed tomographic (CT) scanning and 3D printing can maximize the surgical outcome on both the functional and esthetic reconstruction levels [6].

Applying these techniques, a custom made prototype skull model that resembles the uninjured state can be obtained before surgery; therefore, the surgeon can plan and execute the surgery using a skull model as reference. A synthetic scaffold can be pre-molded to the individual prototype skull model to resemble the anatomic contour before applying it to cover the orbital defects [6].

Our aim in this study is to show the importance and present our experience with three-dimensional virtual planning in solving a variety of acute and chronic clinical deformities within the scope of trauma in the cranio maxillofacial region.

\section{PATIENTS AND METHODS}

Study type: A retrospective analysis of prospectively maintained records of 25 patients with upper and midface fractures. Types of fractures included the: Forehead, orbit, zygomatico-maxillary complex (ZMC) and palatal fractures.

Study setting: The study was performed in 2 different Centers treating Maxillofacial Deformities operated utilizing 3D planning protocols.

Study period: The study was done between September 2015 and August 2018.
A set of inclusion criteria was placed for the cases to be included in the study:

- Complete pre-and post-operative records.

- A full documentation of the follow-up period had to be available.

- Records of the planning protocols and techniques available.

- A follow-up period that exceeds 6 months.

Preoperative clinical, radiological assessment and planning:

All patient had facial deformities either due to acute trauma or chronic mal-united fractures. The etiology of trauma was due to road traffic accidents. Patient with acute trauma were primarily surveyed in the emergency department and further management was done afterward. The surgical plane was individualized according to type of trauma. Indication for surgical intervention was based on priority of correction of the functional and aesthetic by proper skeletal reduction and stability.

The patient age, functional deficit, fracture site, number, type, deformities all are factors to be considered. Patients or their parents were counseled and clearly discussed the nature of the trauma, limitations of surgical outcomes, and the possible secondary procedures to be carried afterward. Involvement of other specialties when needed is of utmost importance e.g.: Ophthalmological, ear nose and throat, and orthodontists according to each patient clinical condition.

Multi-slice cut scan performed. The DICOM images were imported to Mimics 10 software (Materialise NV, Inc, Leuven, Belgium). Thresholding and segmentation was performed to allocate the fractured facial bones. The images were manipulated to prepare a mirror image from the normal uninjured side. An intact full skull image was prepared. The data were exported as STL model for printing a stereo-lithographic model. The hardware to be applied is then applied to the model in order to act as a guide for proper reduction and fixation Fig. (1). 

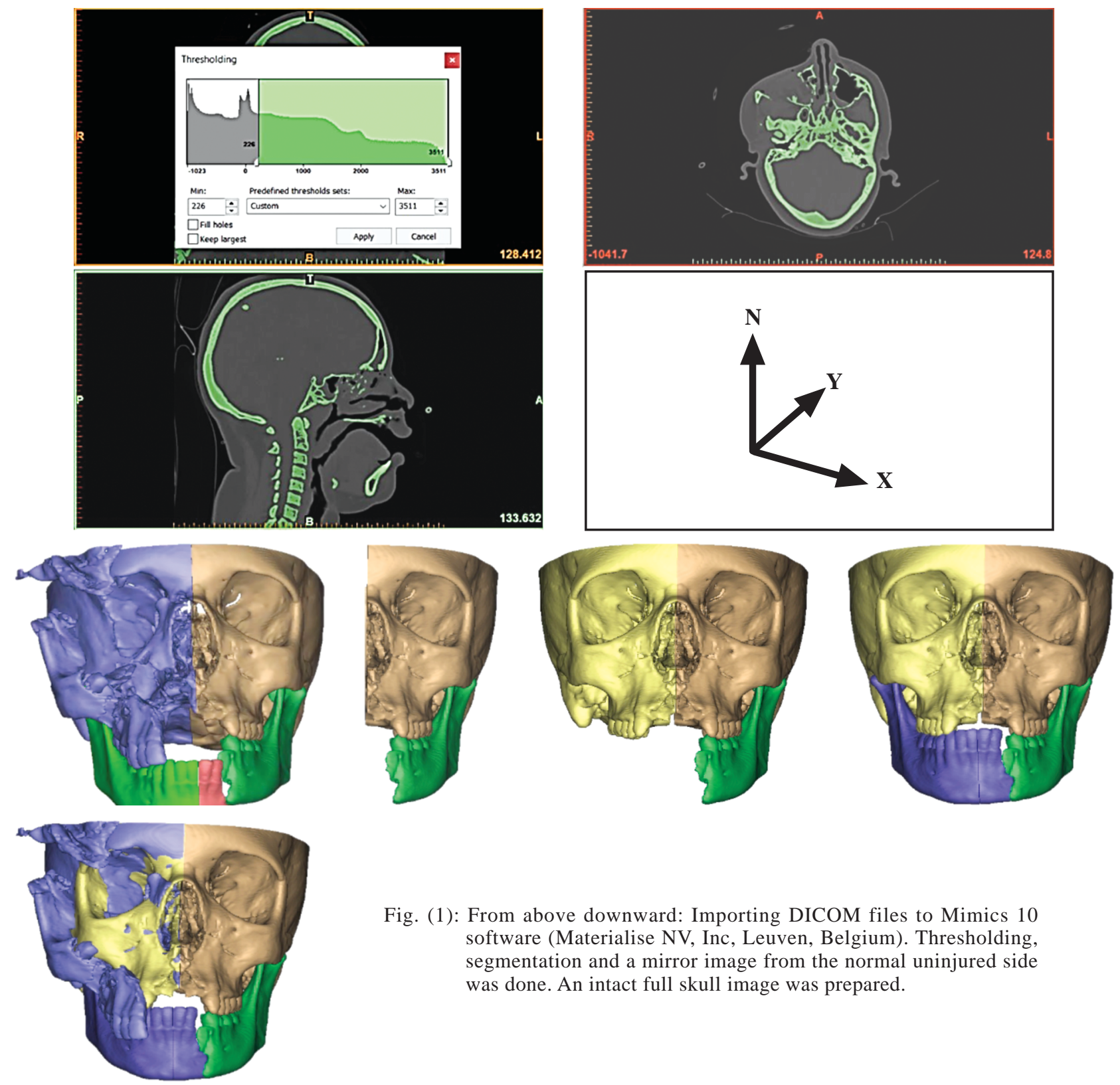

Fig. (1): From above downward: Importing DICOM files to Mimics 10 software (Materialise NV, Inc, Leuven, Belgium). Thresholding, segmentation and a mirror image from the normal uninjured side was done. An intact full skull image was prepared.

\section{Surgical details:}

All surgeries were performed under general anesthesia. Broad spectrum antibiotics were administered. In acute cases, adequate surgical debridement and through wash with and removal of foreign bodies i.e. gravel was done prior to sterilization.

The surgical technique used were tailored to each patient clinical condition:

1- Different surgical incisions adopted: Upper blepharoplasty, subcilliary, upper sulcus and lower sulcus incisions, coronal and current lacerations were used.
2- Centric occlusion is achieved using upper and lower arch bars and maxillary mandibular fixation was done if needed.

3- Identifying and Preserving important structures i.e.: Levator palpebrae superioris muscle, medial and lateral canthi, lose tooth, etc.

4- Guided with the 3 dimensional printed skull, skeletal stabilization i.e. open reduction internal fixation using different shapes of mini plate and screws and or titanium mesh according to the type, site and degree of comminusion. In such cases where exposure of the supraorbital bar or the roof of the orbit was needed, a bicoronal skin incision was employed with scalp 
reflection (preserving supra-orbital neurovascular bundle) until the bar is exposed. A limited frontal craniotomy is done with gentle dissection of the frontal dura to expose the floor of the anterior cranial fossa (i.e. orbital roofs) for reconstruction.

5- Identifying injured tissue layers and the repair was done from deep to superficial. Repair of any injured mucosal lining, Submuscular aponeurotic system, varies muscle injuries using 3/0 and $4 / 0$ vicryle either in interrupted or continues type.

6- Application of suction drains if needed, followed by skin closure using $5 / 0$ and $6 / 0$ proline stitches.

\section{Post-operative care:}

Light compression dressing were applied to all patients. Patients laid down in semi sitting position. Cold fomentation for the first 48 hours followed by hot fomentation up to two weeks. Intravenous antibiotics administered till the drain is removed, shifting to oral route for one week. Drains removed when it's minimal amount (less than 30cc). Dressing is done at the $3^{\text {rd }}$ postoperative day. The skin stitches were removed at the $5^{\text {th }}$ or $7^{\text {th }}$ postoperative days. All patients instructed to follow up in the outpatient clinic at one, three and six months afterward. Patients was informed about possible progress, and possible outcome which might need 2ry procedures.

\section{Post-operative clinical and radiological assess- ment:}

All patients were examined for the aesthetic outcome, presence of complications and multi slice cut scan was performed, the DICOM images were transferred to mimics software (MaterialiseNV, Inc, Leuven, Belgium). Deviations from normal will be quantified using a color map. The analysis statistics will be described applying the unsigned mode for point to point comparison. The absolute mean and standard deviation was reported.

Patients demographic, history, physical and clinical examination, clinical photographs and surgical procedures (number and type) and complications were collected. All patients or their parents signed an informed consent to be included in the study.

\section{RESULTS}

25 patients were retrospectively recruited. Mean age was 33.5 years (range 13-59), male: female ratio $=2.6: 1(18: 7)$. Eleven patients had acute injuries (44\%) while the remaining 14 patients $(56 \%)$ had chronic (malunited) fractures (Diagram 1).

The ZMC was involved alone in 7 patients (28\%), and along with the orbit in the remaining 18 patients $(72 \%)$. Of the latter group, 8 patients had additional frontal bone fractures $(32 \%$ of the whole cohort). Not all patients with orbital fractures had repair by titanium mesh, leaving 4 patients without orbital meshes implanted. Such decision was based on the intraoperative findings. This is contrary to the patients with forehead fractures, where all $(n=7)$ but one had titanium mesh fixation. Maxillary titanium meshes were placed in 6 patients (24\%). (Diagrams 2,3,4). Fixation was a 3-point fixation in the majority of the patients, $56 \%(n=14)$ and 4-point fixation in $44 \%(\mathrm{n}=11)$.

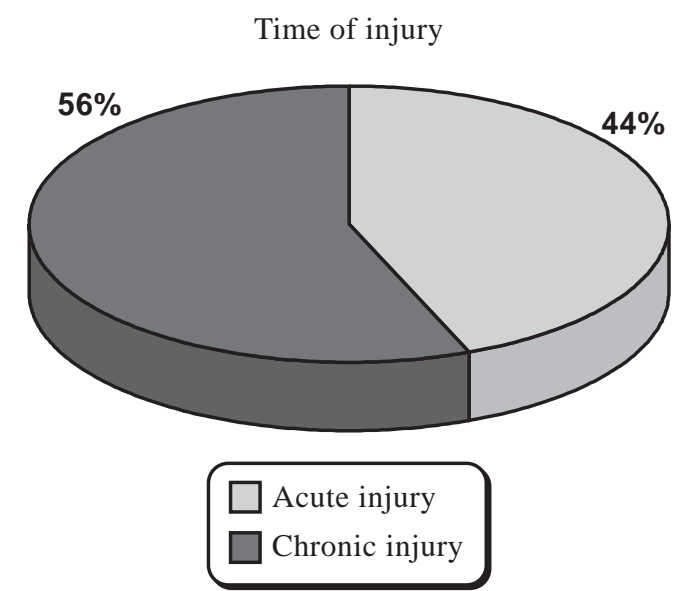

Diagram (1): Showing patients distribution as acute and chronic deformities.

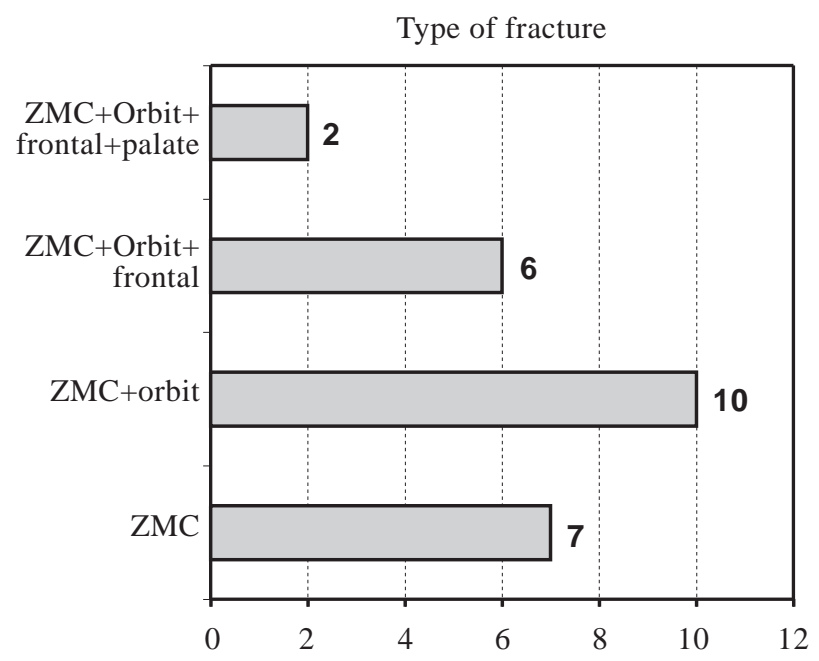

Diagram (2): Showing the distribution of different types of cranio-maxillofacial fractures. 


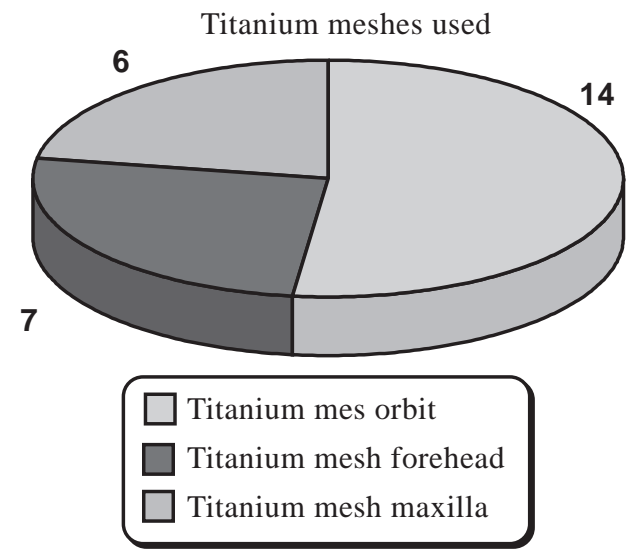

Diagram (3): Distribution of the titanium mesh used according to the site.

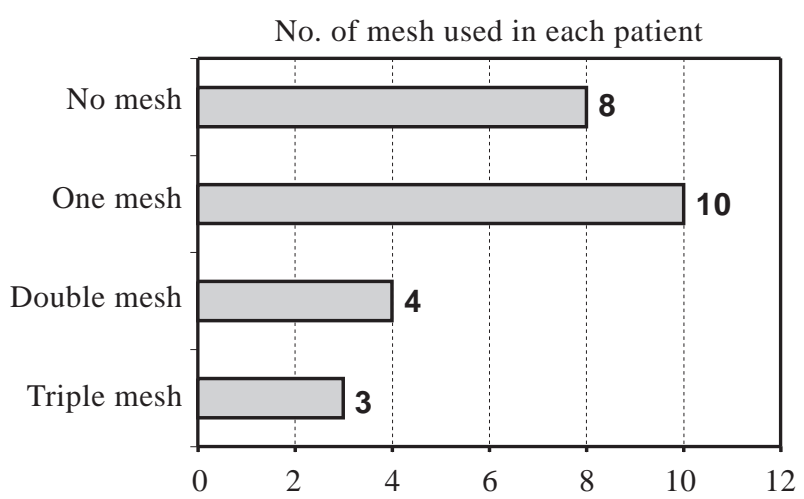

Diagram (4): Showing the number of titanium mesh used in each patient.

\section{Statistical analysis:}

Statistical analysis was performed using IBM SPSS Statistics Version 2.1 for Windows. Data was presented as mean and standard deviation (SD). The significance level was set at $p \leq 0.05$. Kolmogorov-Smirnov and Shapiro-Wilk tests were used to assess data normality. Data showed nonnormal distribution, so Mann-Whitney test was performed to compare between healthy and fixed orbit volumes.

There was no statistically significant difference between healthy and fixed orbits volumes (Table 1 \& Diagram 5). Complex cranio-maxillofacial fractures were assessed using the mirrored reconstructed images and the post-operative fixation images. The overall deviation mean from normal was calculated to be $1.41 \pm 0.15 \mathrm{~mm}$. The amount of deviation measured for each patient is presented in Table (2) and Diagram (6).

Table (1): Mean \pm SD of healthy and fixed orbit volume measurements $\left(\mathrm{mm}^{3}\right)$.

\begin{tabular}{lllr}
\hline & Healthy orbit & Fixed orbit & $p$-value \\
\hline $\begin{array}{l}\text { Volume } \\
\left(\mathrm{mm}^{3}\right)\end{array}$ & $33174.14 \pm$ & $33525.69 \pm$ & 0.580 \\
\hline
\end{tabular}

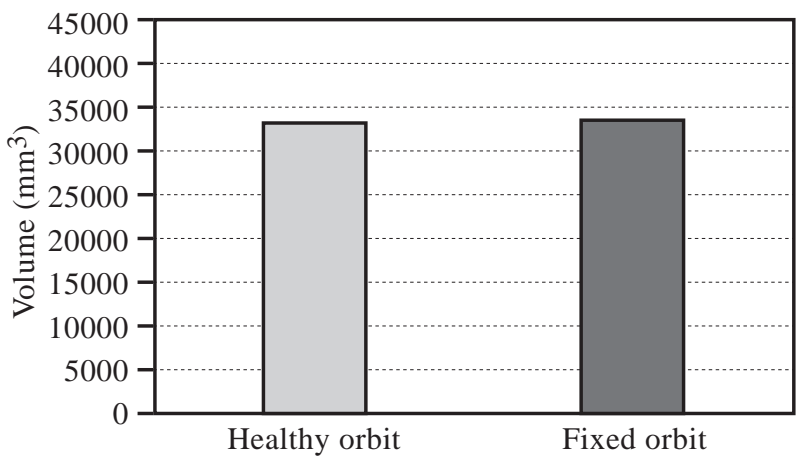

Diagram (5): Volume measurements $\left(\mathrm{mm}^{3}\right)$ of healthy and fixed orbits.

Table (2): Distance (deviation) away from the mirrored intact side (the Control): Measured in ( $\mathrm{mm}$ ).

\begin{tabular}{lcc}
\hline Patient & $\begin{array}{c}\text { Distance (deviation) away from } \\
\text { the mirrored intact side } \\
\text { (the Control) }\end{array}$ & $\begin{array}{c}\text { Standard } \\
\text { deviation }\end{array}$ \\
\hline 1 & 1.3008 & 1.208 \\
2 & 1.478 & 1.1771 \\
3 & 1.409 & 1.3493 \\
4 & 1.3046 & 1.256 \\
5 & 1.508 & 1.1265 \\
6 & 1.784 & 1.637 \\
7 & 1.276 & 1.158 \\
8 & 1.56 & 1.439 \\
9 & 1.125 & 1.148 \\
10 & 1.367 & 1.256 \\
11 & 1.374 & 1.272 \\
12 & 1.3002 & 1.203 \\
13 & 1.458 & 1.324 \\
14 & 1.362 & 1.235 \\
15 & 1.3579 & 1.246 \\
16 & 1.3046 & 1.256 \\
17 & 1.508 & 1.1265 \\
18 & 1.784 & 1.637 \\
19 & 1.276 & 1.158 \\
20 & 1.56 & 1.439 \\
21 & 1.567 & 1.256 \\
22 & 1.374 & 1.272 \\
23 & 1.3002 & 1.203 \\
24 & 1.358 & 1.324 \\
25 & 1.362 & 1.235 \\
\hline
\end{tabular}

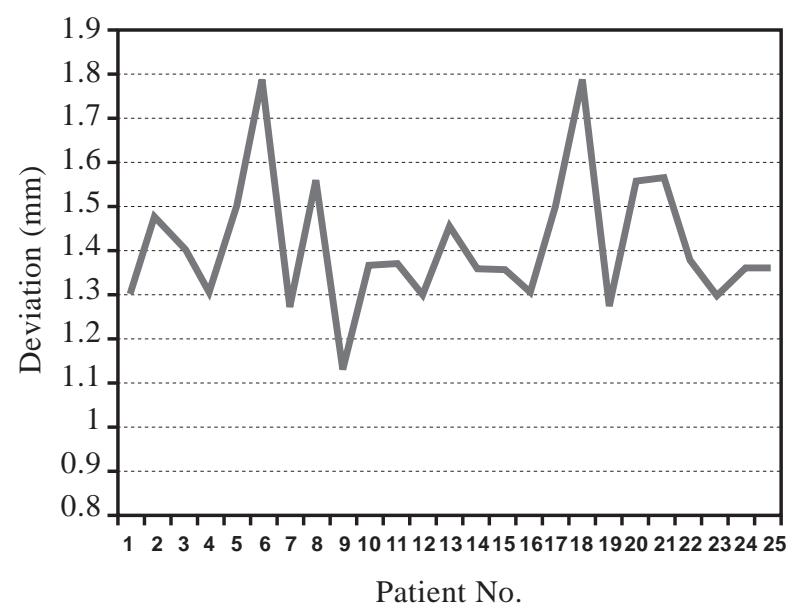

Diagram (6): Deviation ( $\mathrm{mm}$ ) from the contralateral side for each patient. 
Their no significant complication recorded other than soft tissue atrophy over the frontal and periorbital region in two patients which will need

Case (1)
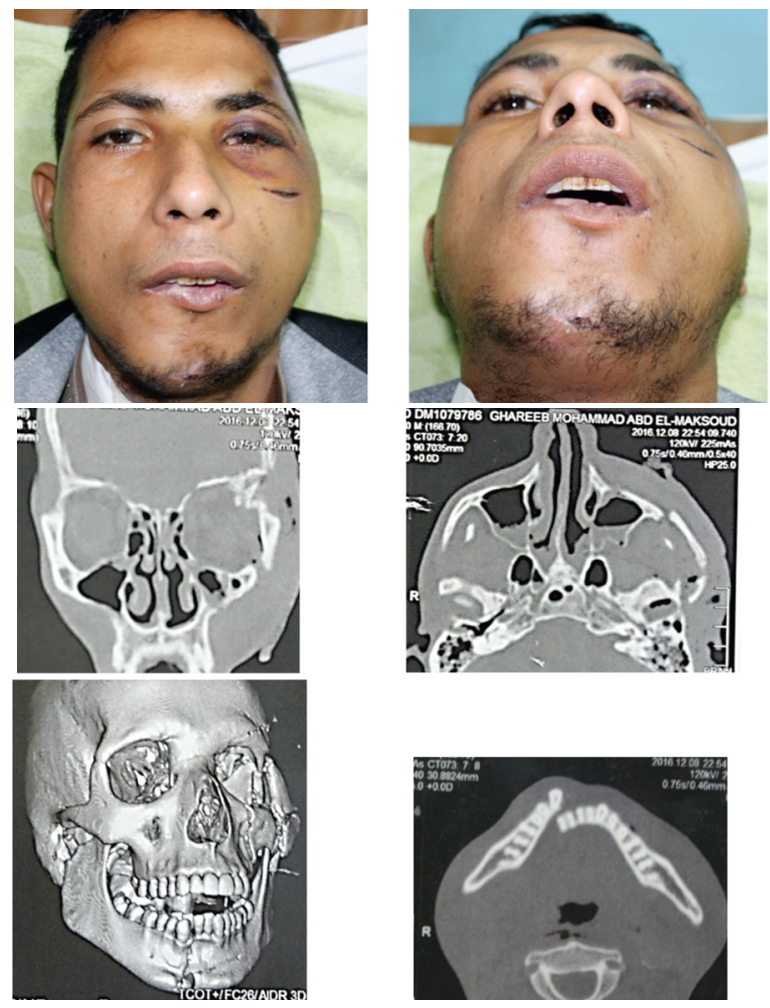

touch up procedure in the form of fat injection. Fig. (2) till (21) show some of the cases included in the study.

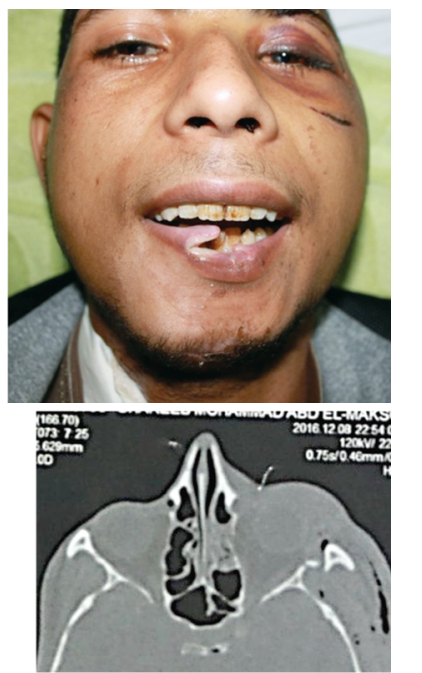

Fig. (2): Male patients 19 years old with tetrapod fracture post road traffic accident. (upper raw): Preoperative photos. (middle raw and lower raw): Pre-operative CT scan views.
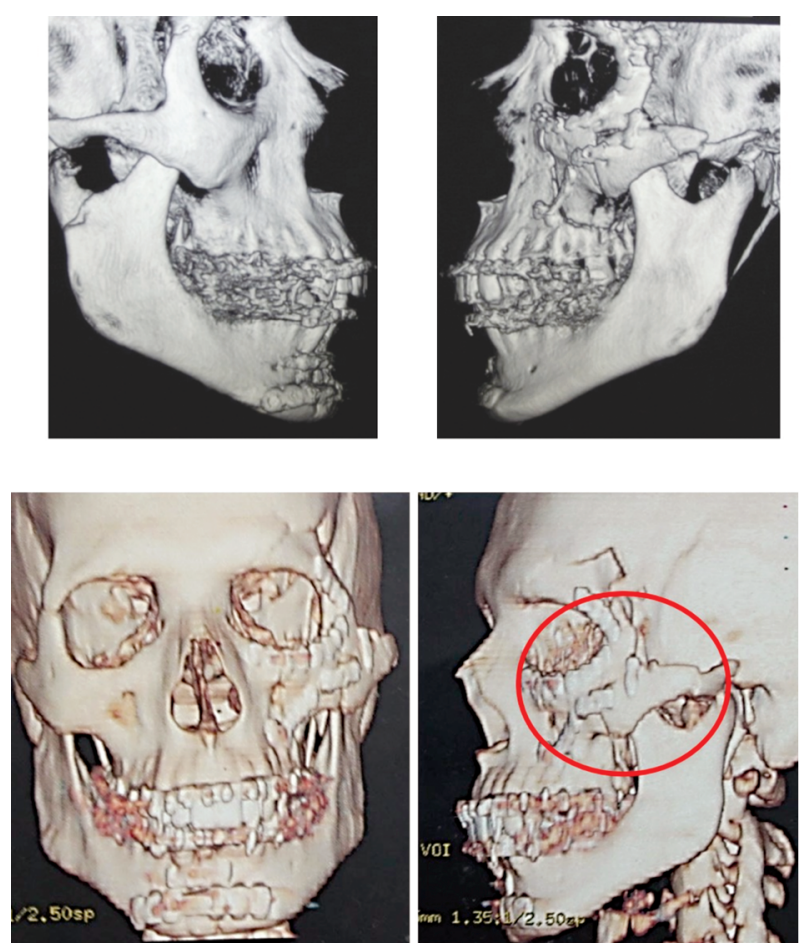

Fig. (3): (Upper raw): Post-operative CT scan photos. (Lower raw): CT scan views showing mal alignment of the zygomatic arch.

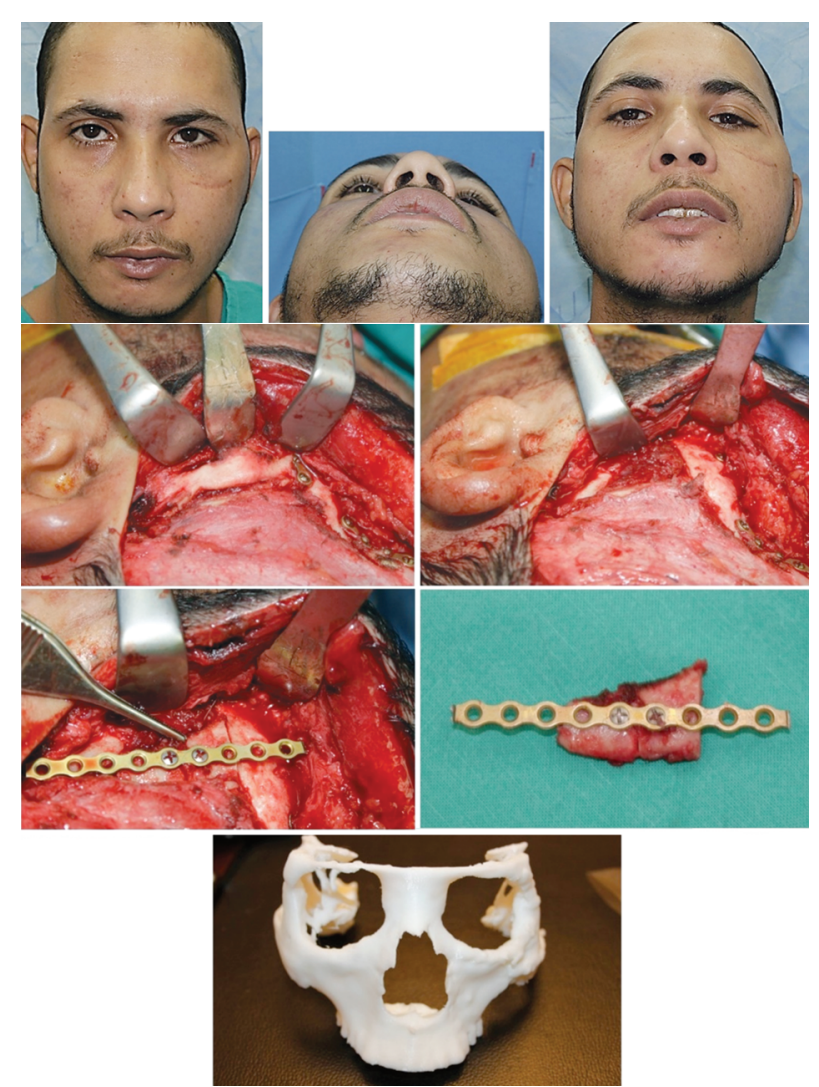

Fig. (4): ( $1^{\mathrm{St}}$ raw): Post-operative photos of the patient showing depressed and medially rotated left zygoma. ( $2^{\text {nd }}$ raw and $3^{\text {rd }}$ raw): Intra-operative photos showing the exposure of the zygomatic arch, corrective osteotomy and re-fixation. CT scan views. (4 ${ }^{\text {th }}$ raw): The printed three dimension model. 
Fig. (5): (Upper and middle raw): Corrective osteotomy of the zygomatic arch, frontozygomatic, orbital rim and body of zygoma, post-operative CT scan. (Lower raw): 9 months post-operative photo.
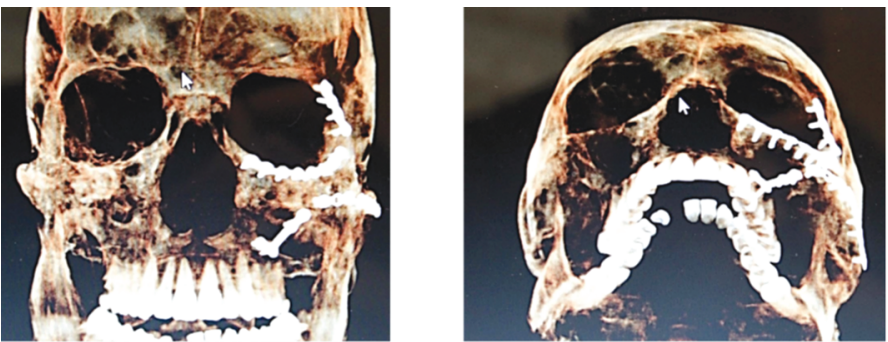

(1)
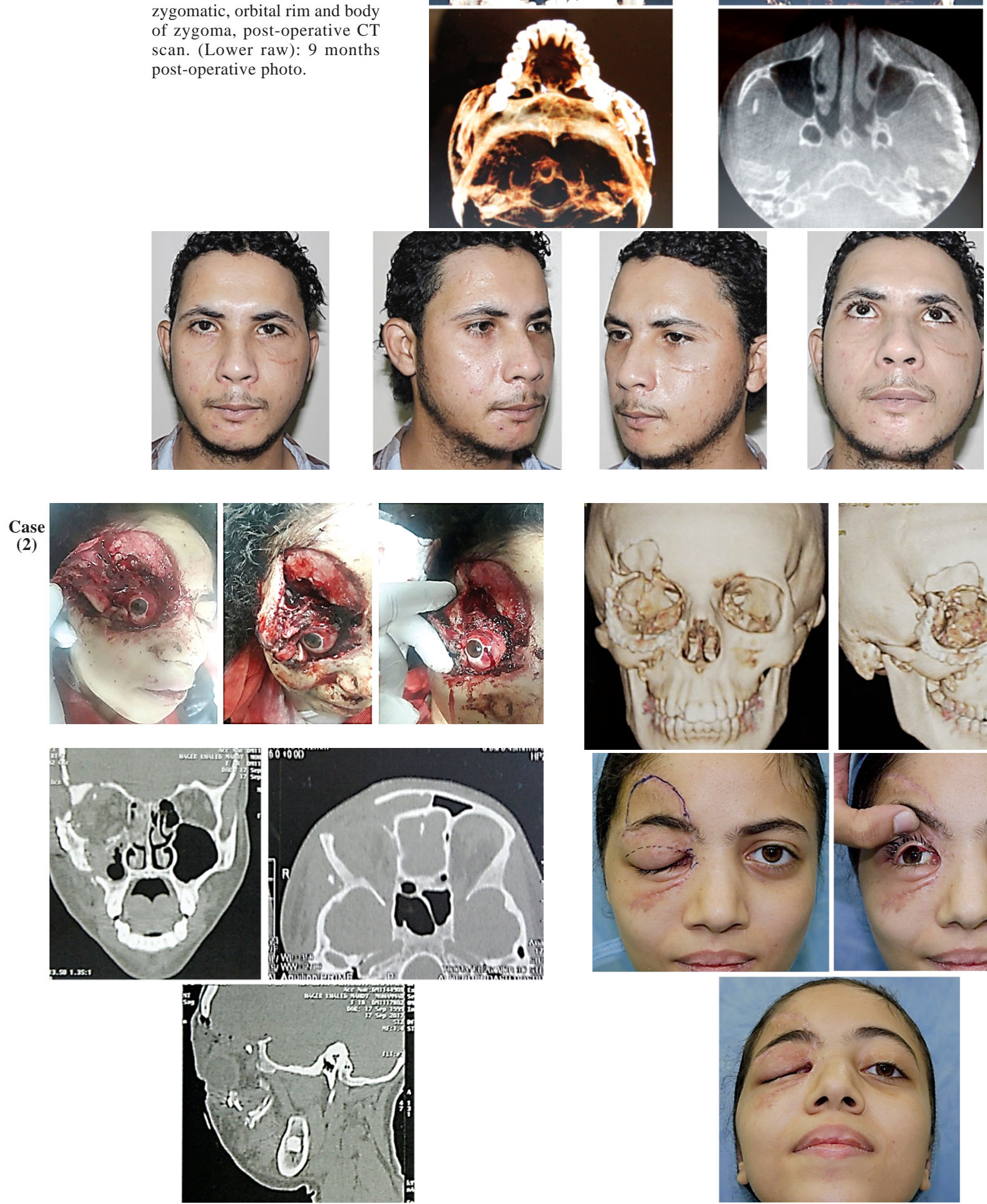

Fig. (6): Female patient 18 years old post road traffic accident with comminuted frontal, orbital floor and right zygoma, associated with tissue injury (upper raw): Preoperative photos, showing the severity of tissue injury. (Middle and lower raw): Preoperative CT scan.
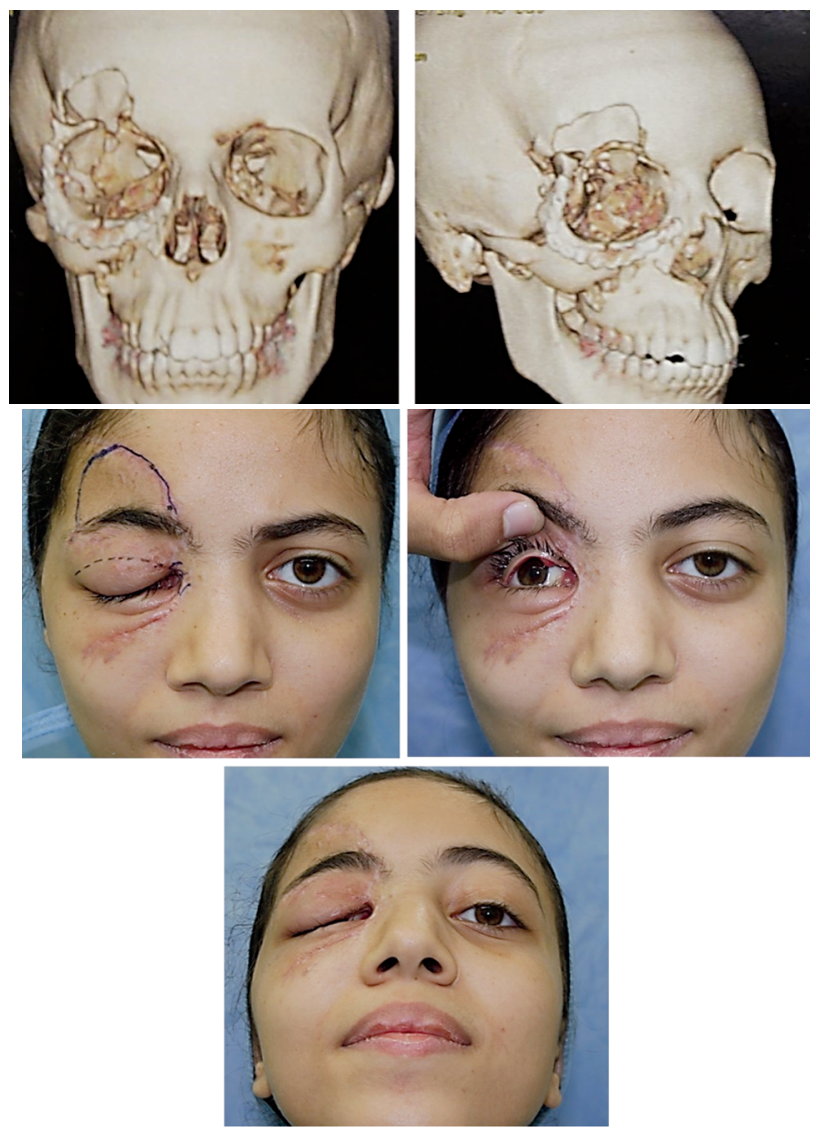

Fig. (7): (Upper raw): $1^{\text {st }}$ stage pot-operative cut scan, showing fixation of the right zygoma without reconstruction of the right frontal bone and roof of the orbit. (Middle and lower raw): Early postoperative photos. 

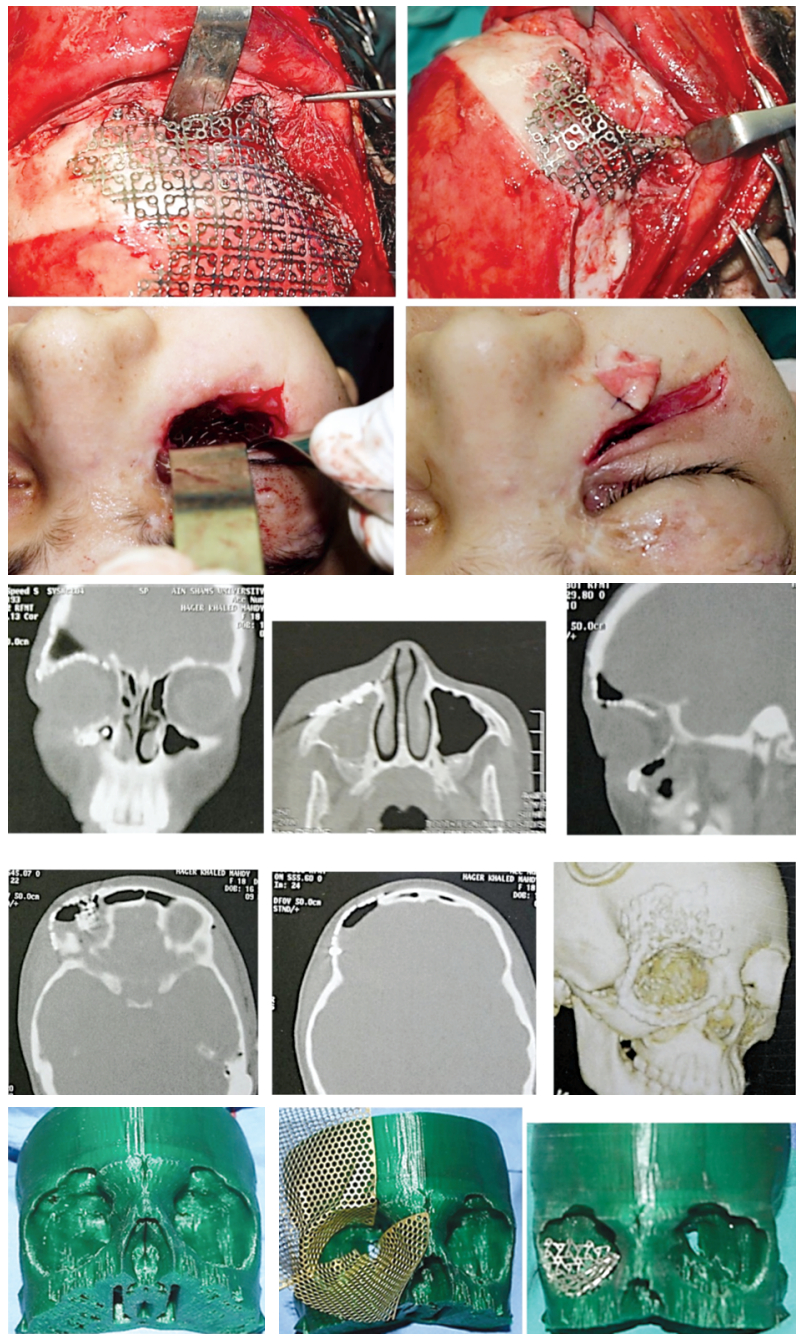

Fig. (8): ( $1^{\text {st }}$ and $2^{\text {nd }}$ raw): Intra-operative photos, showing reconstruction of the right frontal bone, roof and floor of the orbit, fronto zygomatic region using titanium mesh and conchal cartilage graft. ( $3^{\text {rd }}$ and $4^{\text {th }}$ raw): Early post-operative photos. $\left(5^{\text {th }}\right.$ raw): The printed three dimension model and intra-operative adaptation.

Case (3)
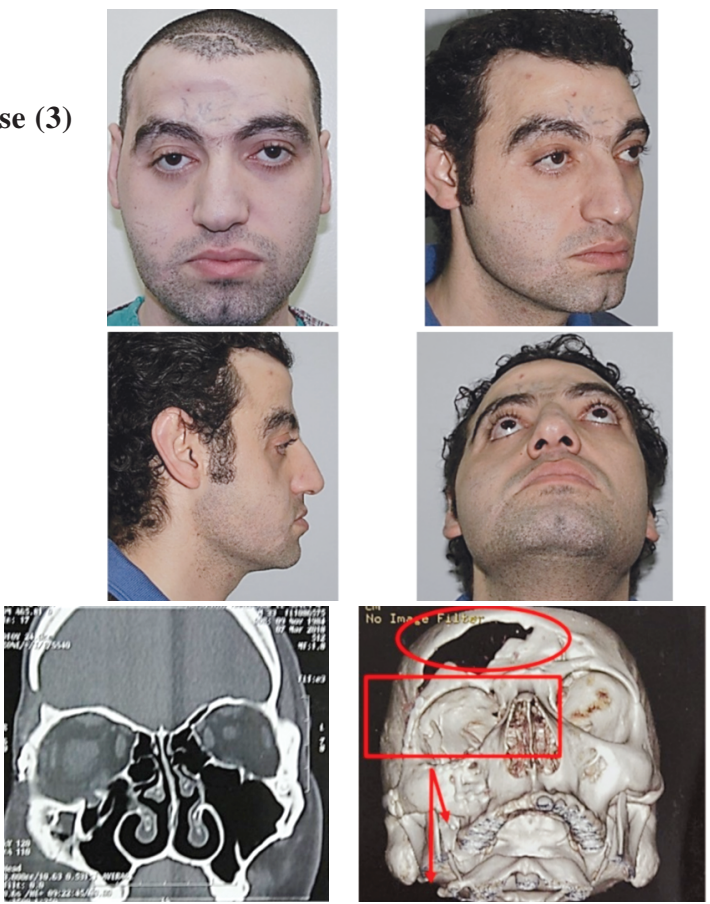

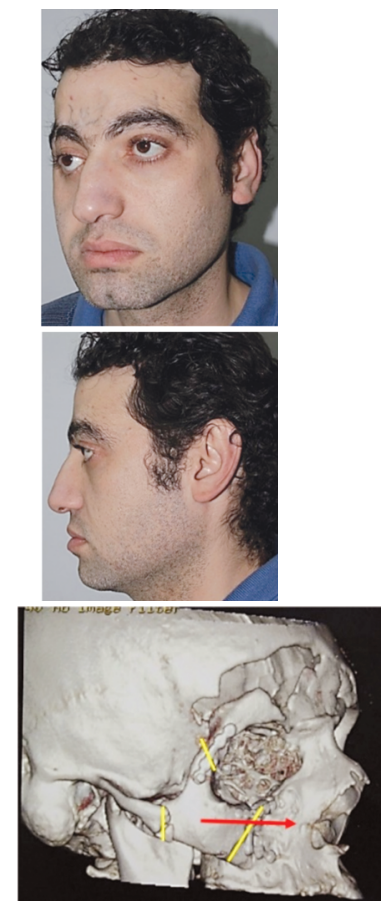

Fig. (10): 32 years old male patient with chronic post traumatic cranio maxillofacial deformity. (Upper and middle raw): Preoperative photos showing the severity of the deformity at the frontal, orbital and ZMC region. (Lower raw): Preoperative CT scans with red circle and arrow showing the frontal defect and the malposition right ZMC. Yellow arrow shows the future corrective osteotomy sites. 

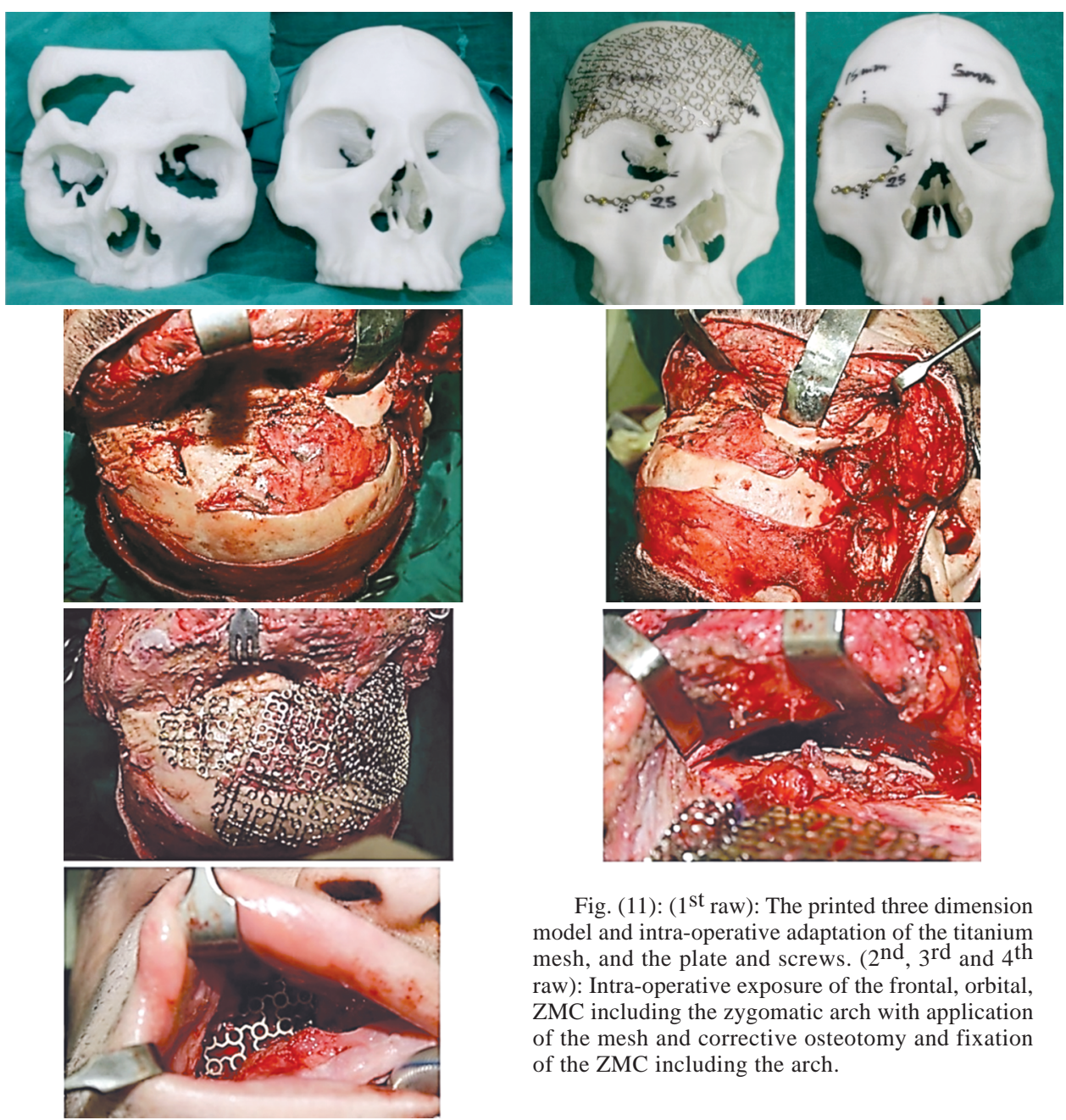

Fig. (11): ( $1^{\text {st }}$ raw): The printed three dimension model and intra-operative adaptation of the titanium mesh, and the plate and screws. (2nd, $3^{\text {rd }}$ and $4^{\text {th }}$ raw): Intra-operative exposure of the frontal, orbital, ZMC including the zygomatic arch with application of the mesh and corrective osteotomy and fixation of the ZMC including the arch

Fig. (12): (Upper raw): Post-operative Cut Scan showing the tetra pod fixation of the ZMC and reconstruction of the Frontoorbital and maxillary region with titanium mesh. (Lower Raw): Post-operative photos.
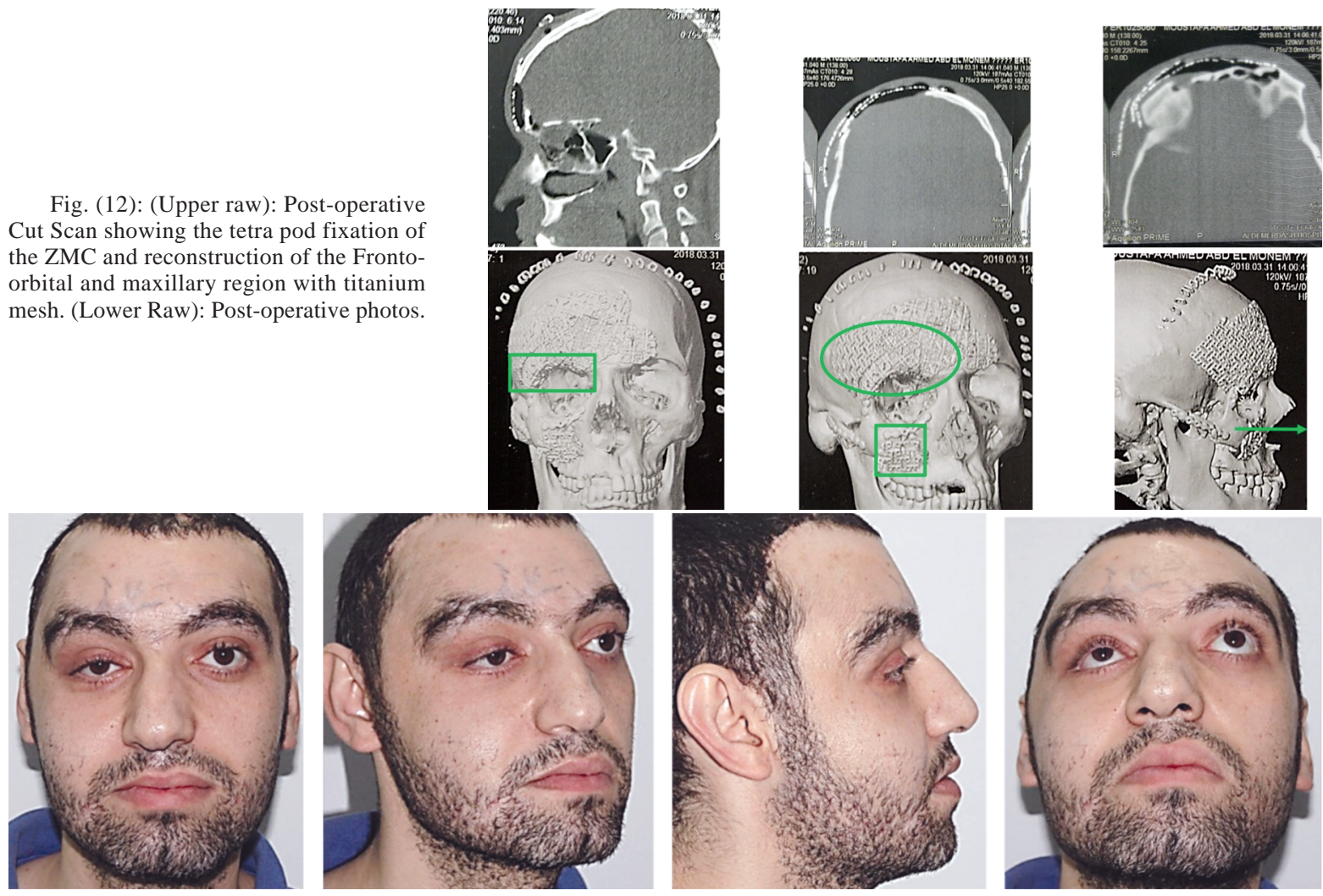

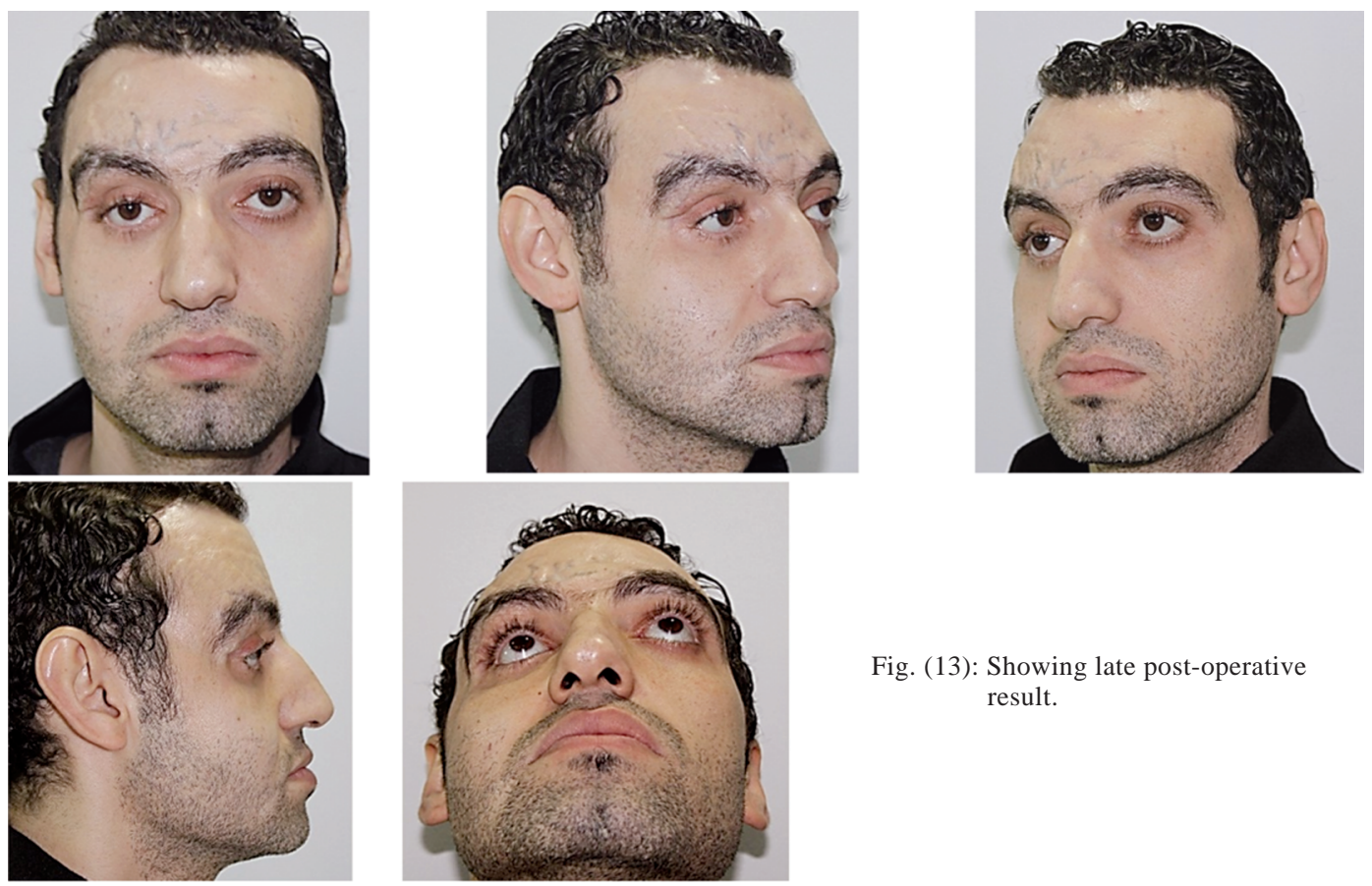

Fig. (13): Showing late post-operative result.

Case (4)
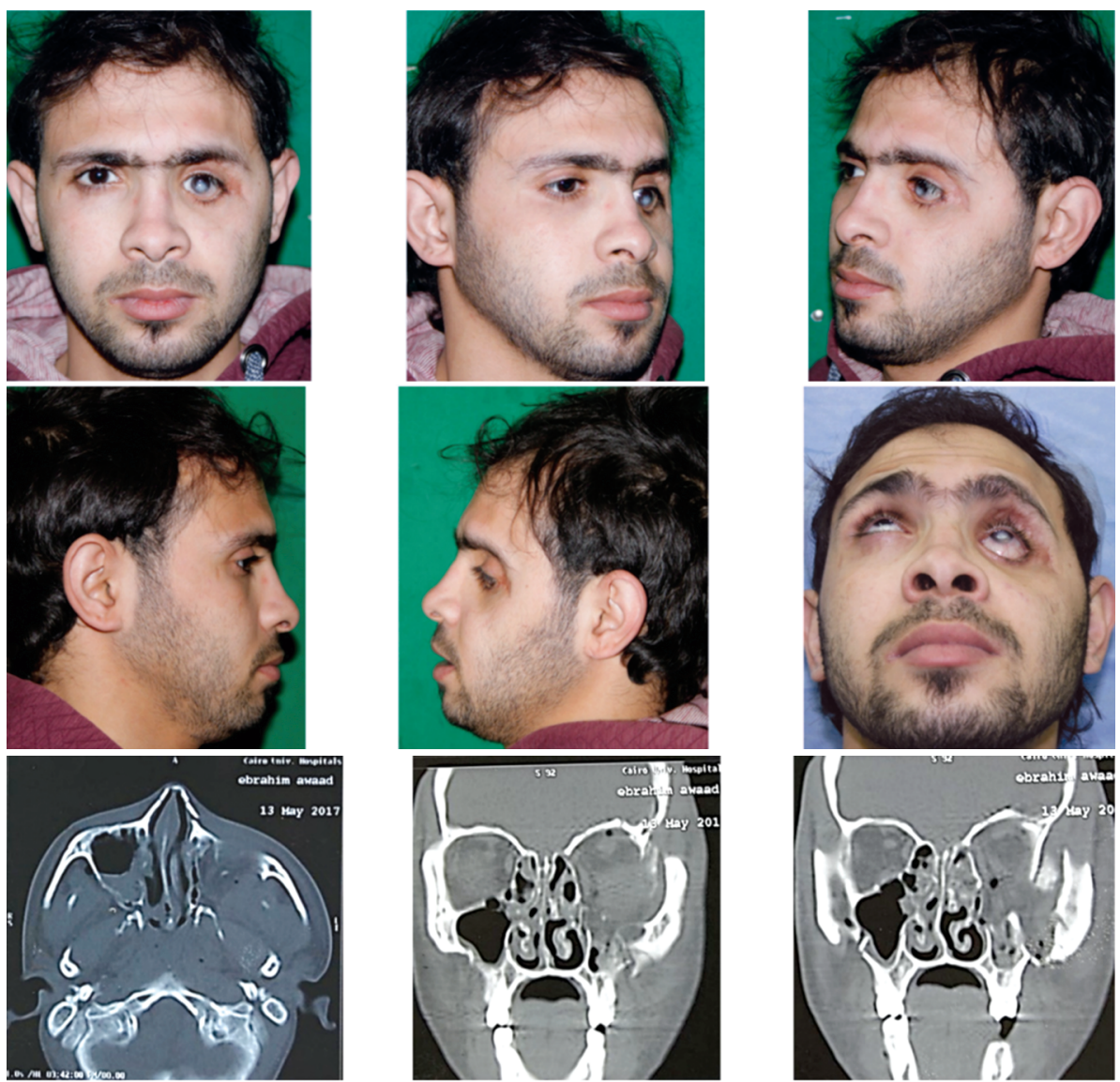

Fig. (14): 29 years old male patient with chronic post traumatic cranio maxillofacial deformity of the left ZMC complex and frontal bone complicated with corneal ulcer and opacity of the left cornea. (Upper and middle raw): Preoperative photos showing the severity of the deformity at the ZMC and frontal region. (Lower raw): Pre trauma CT scans.

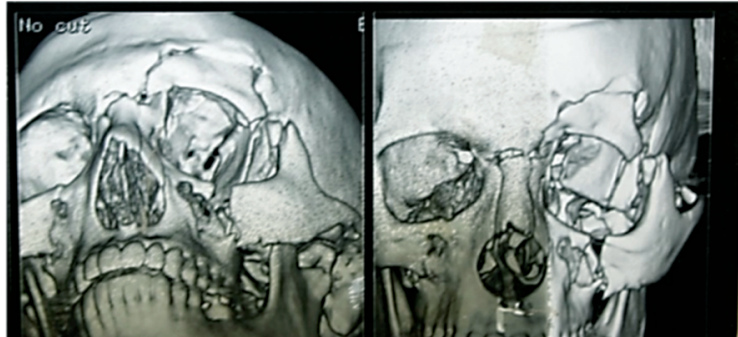



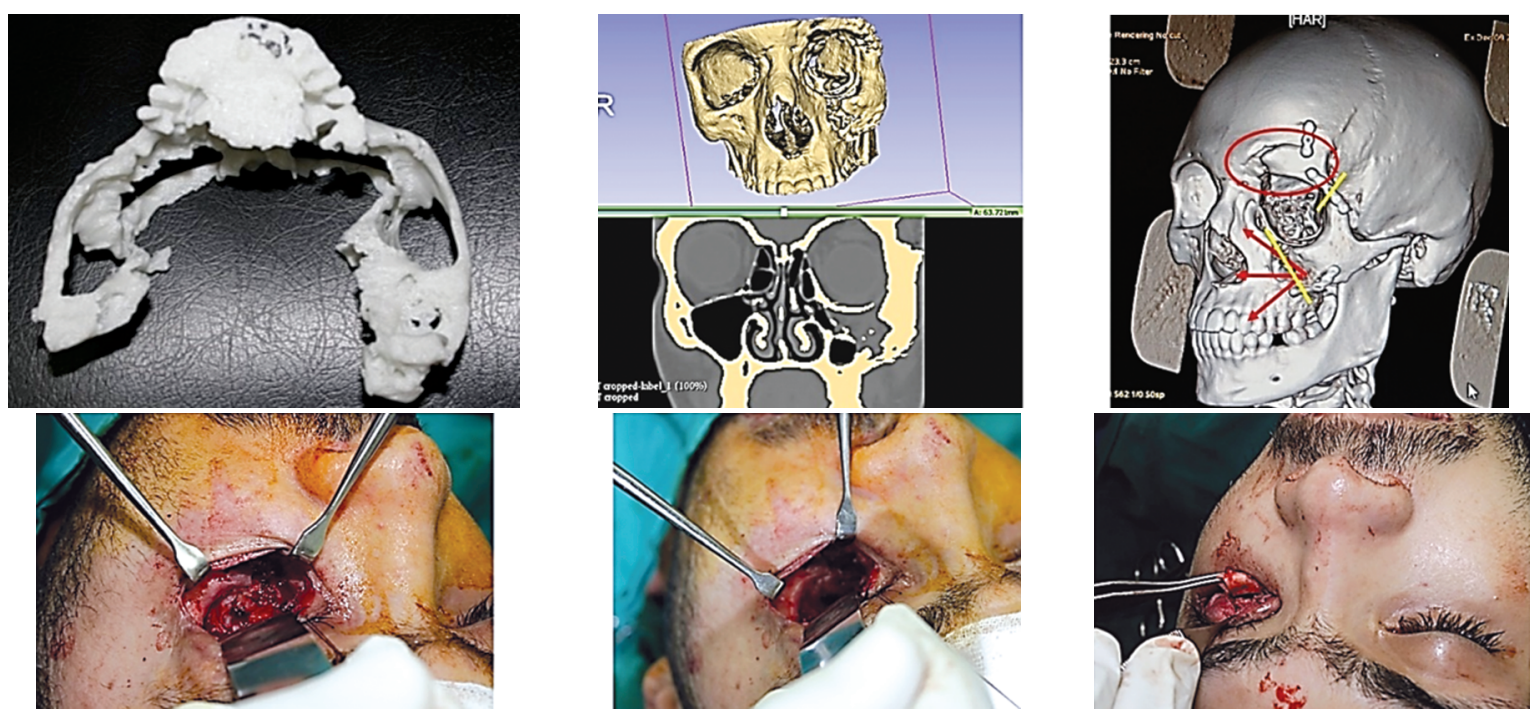

Fig. (15): (Upper raw): Preoperative planning and three dimension model with medially rotated and impacted right zygoma. 3 dimension cut scan with red circle and arrows showing the region to be corrected, yellow arrows indicate the line of osteotomy. (Lower raw): Intraoperative photos showing the degree of displacement of the orbital rim fracture and application of conchal cartilage augmenting of the orbital floor.
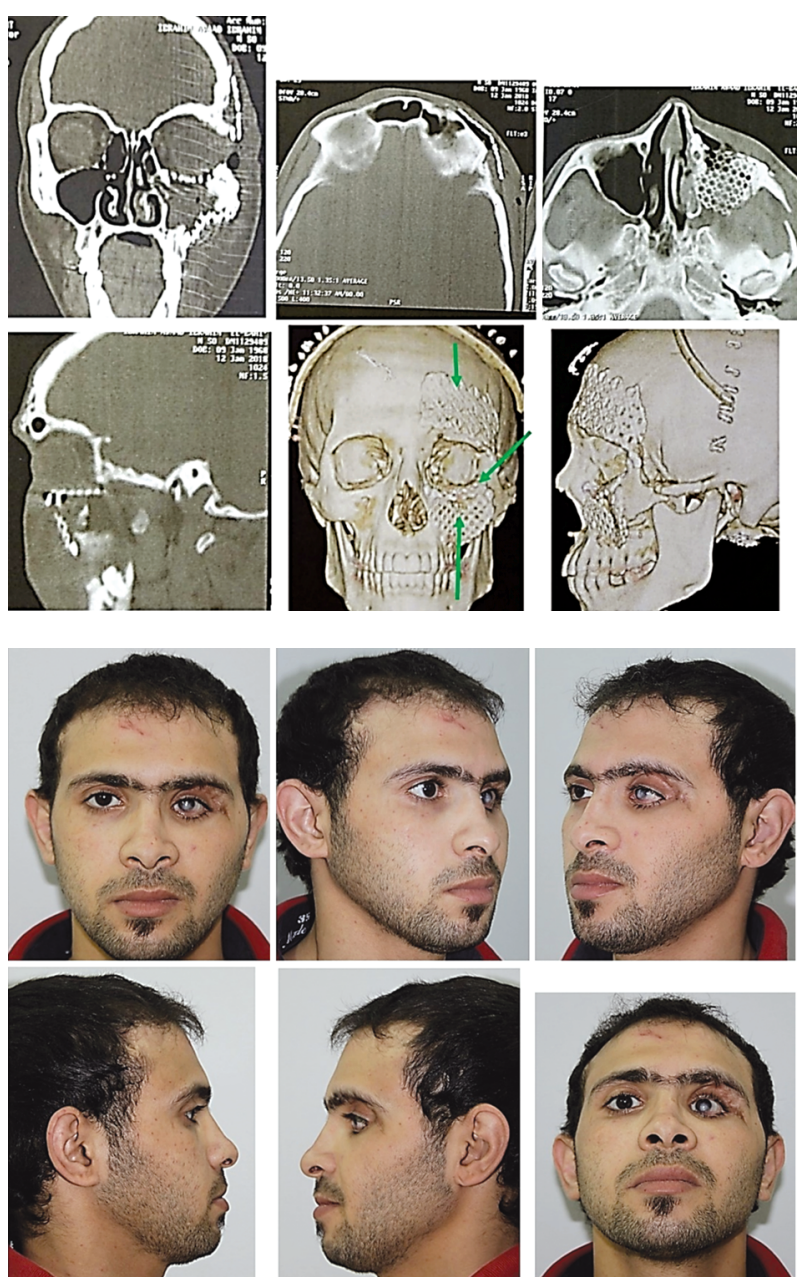

Fig. (16): (1 $1^{\text {st }}$ and $2^{\text {nd }}$ raw): Post-operative CT scan showing the tripod fixation of the left zygoma in its new position, and reconstruction of the orbital, frontal and maxillary region with titanium mesh. ( $3^{\text {rd }}$ and $4^{\text {th }}$ raw): Late post-operative result.
Case (5)
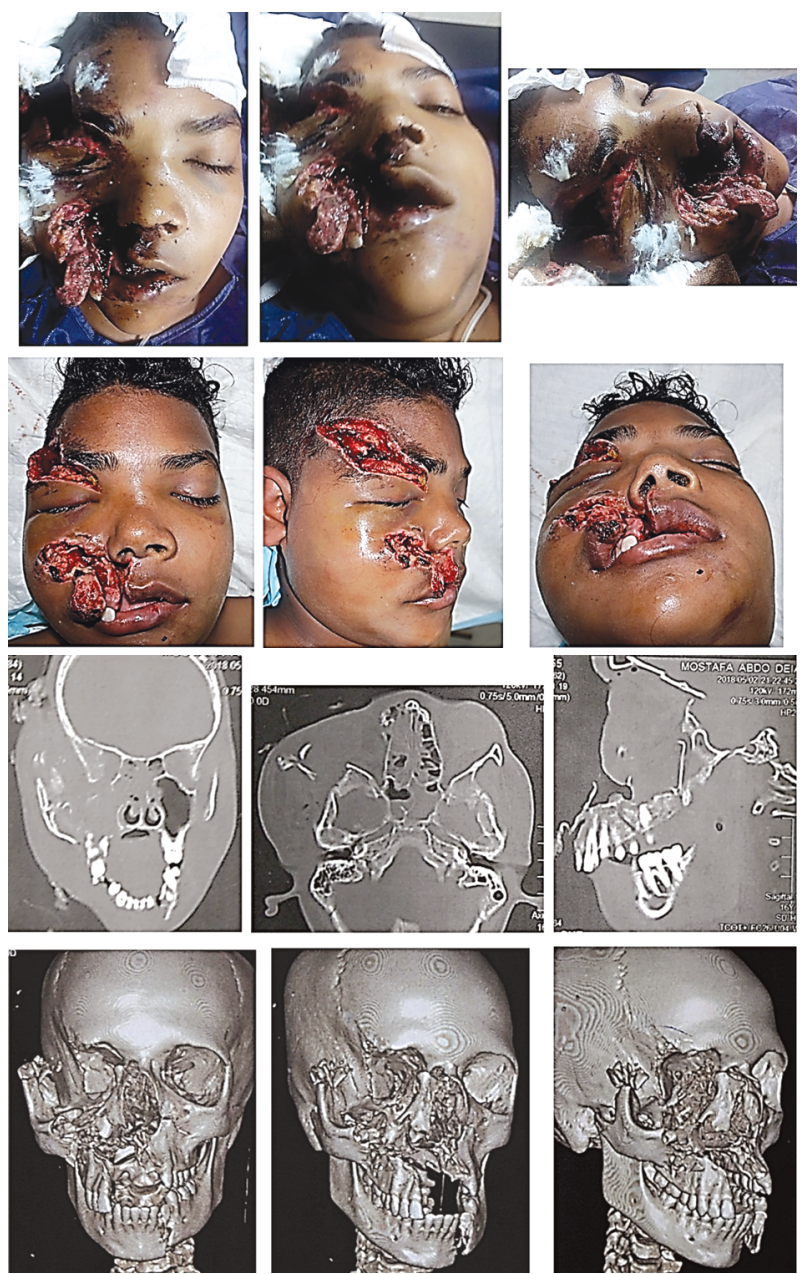

Fig. (17): Male patient 15 years old with acute maxillo facial injury post road traffic accident. ( $1^{\text {st }}$ an $2^{\text {nd }}$ raw): Preoperative photos showing the severity of the facial lacerations and crushing of the frontal orbital and ZMC with down ward displacement and splitting of the right upper maxilla. ( $3^{\text {rd }}$ and $4^{\text {th }}$ raw): Pre-operative CT scan showing crushing and sever displacement of the frontal, orbital, ZMC and palate associated with fracture mandible. 


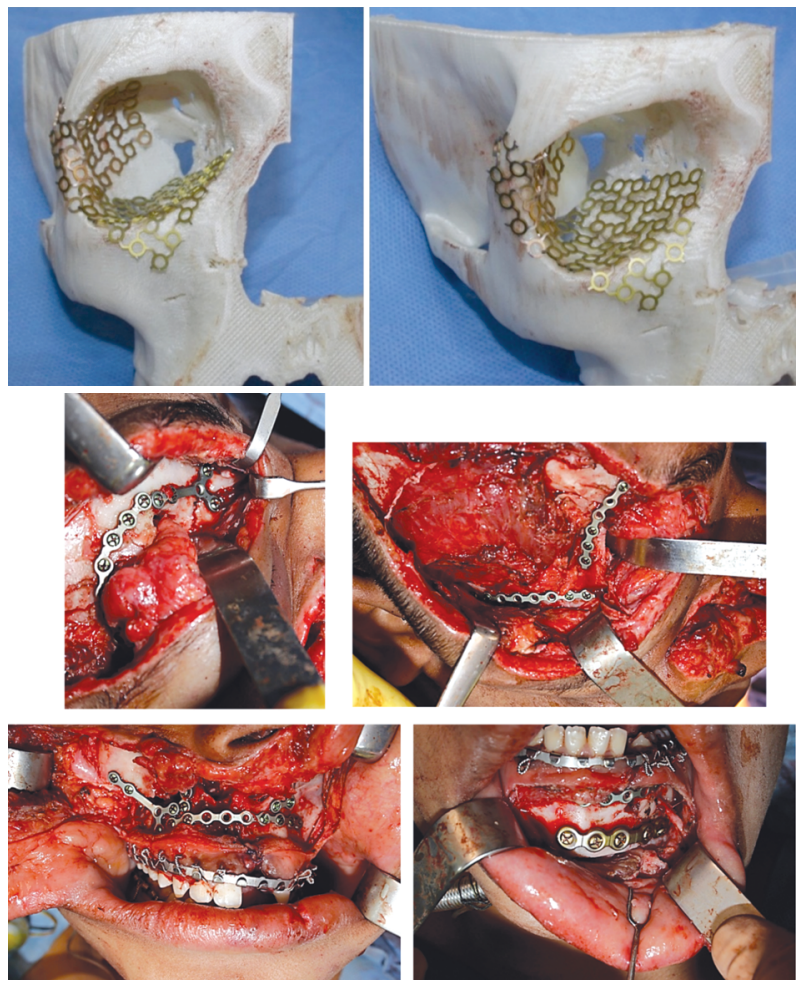

Fig. (18): (1 ${ }^{\text {st }}$ raw): Printed three dimension model and intraoperative bending of the orbital mesh for the floor and lateral orbital regions. ( $2^{\text {nd }}$ and $3^{\text {rd }}$ raw): Intra-operative photos with exposure of the frontal, orbital, zygomatic arch, naso-orbito ethmoid, ZMC and mandible and reconstruction with mini plate and screws.
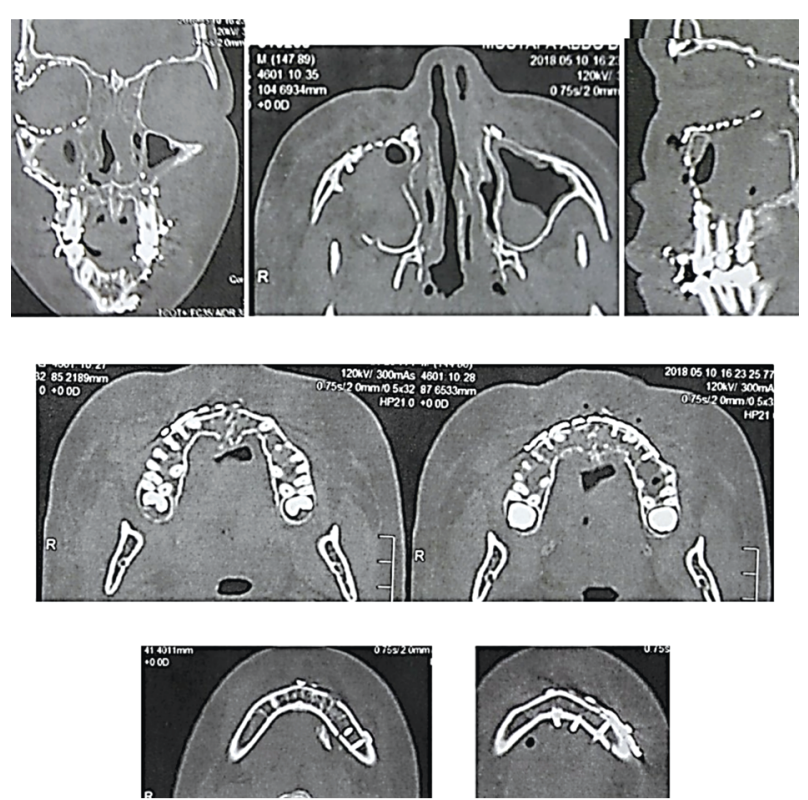
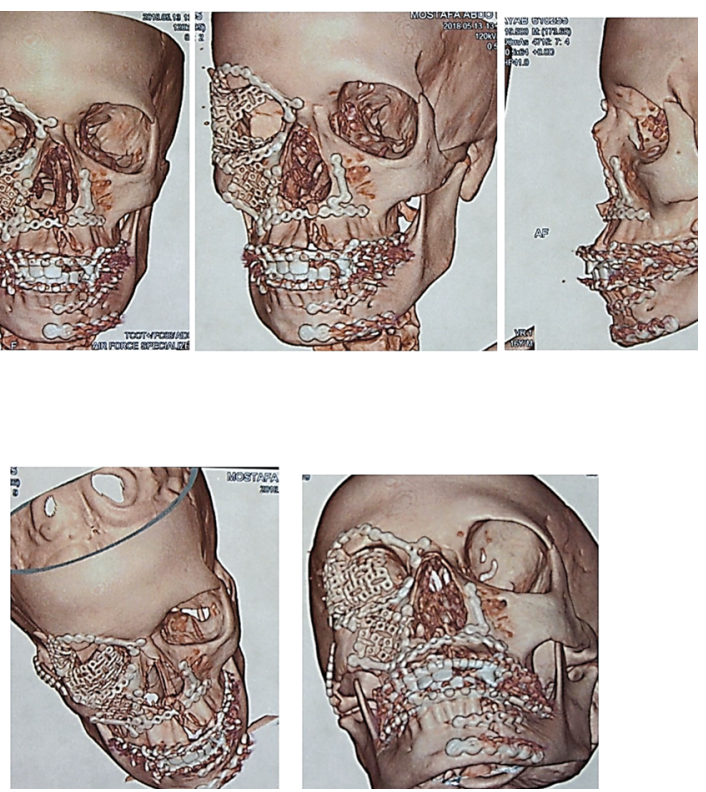

Fig. (20): Showing different views of the post-operative CT scan with adequate reduction of the fractures and application of titanium mesh and the mini plate and screws. 


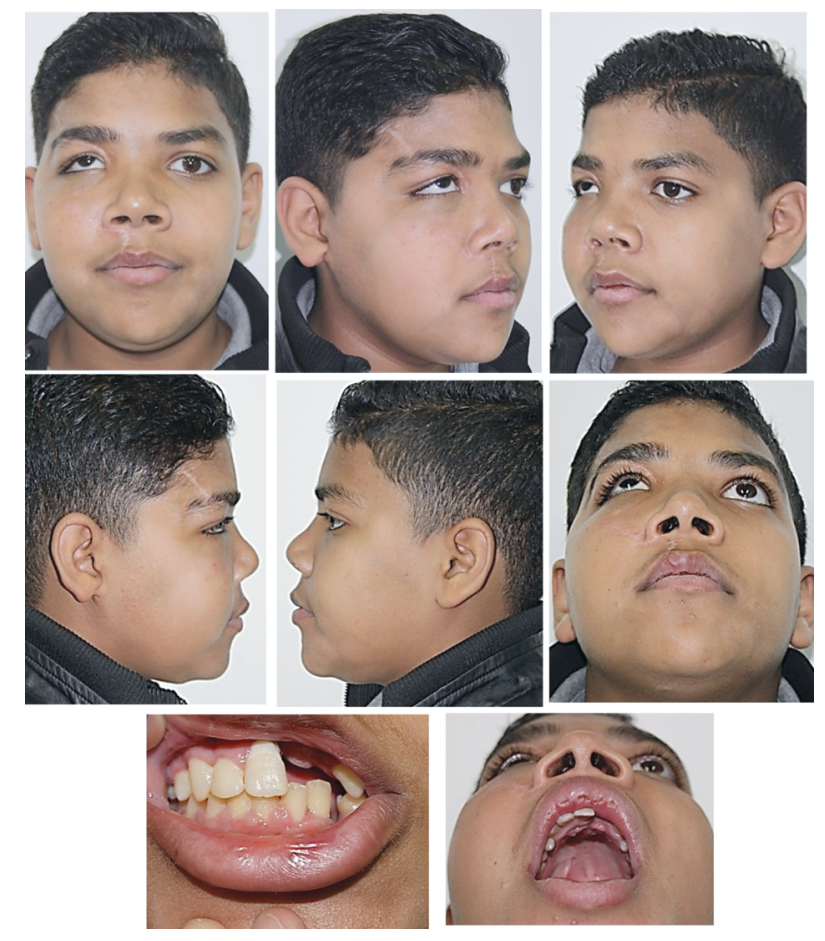

Fig. (21): Late (7 month) post-operative photos with adequate reduction and accepted symmetry. He is scheduled for squint surgery due to post-traumatic weakness of the lateral and inferior rectus muscles.

\section{DISCUSSION}

The complexity of the anatomy and deformities of the cranio-maxillofacial region entail the use of modern technologies like the virtual planning (VP), 3 Dimension modeling (3DM) and 3 dimension printed custom implants (3DPCIs), as these deformities commonly include not only skeletal but also soft tissue components.

These modern technologies are used to facilitate the analysis, diagnose the anatomic difficulty, simulate and orient the surgery, individualized the procedure according to each patient clinical situation, facilitate patient education, anticipate the results, and support the educational field in craniofacial surgery [7-10].

The main indication of 3D virtual planning and printing in cranio-maxillofacial surgery include orthognathic surgery, planning vectors and osteotomies in distraction ostiogenesis, acute and chronic traumatic maxillofacial fractures and deformities, cranioplasty, facial skeletal contouring and augmentation [11-26]. The study included 11 patients with acute and 14 patients with chronic deformities all of which are due to road traffic accidents. 7 patients presented with ZMC fracture, 10 patients presented with ZMC and orbital fractures, 6 patients with ZMC, orbit and frontal bone fractures and lastly 2 patients presented with ZMC, orbit, frontal bone and palatal fractures. All of them managed with open reduction internal fixation with mini plate and screws. Corrective osteotomies was done and preplanned according to the vectors used to regain proper reduction and symmetry. Conchal cartilage graft was used in six patients to correct enopthalmous.

Previous concept and main concerns in planning is the number and site of fixation is the location and the number of fractured buttresses, which need fixation for optimal stability. Applying the concept of 1 point, 2 point, 3 point and 4 points of fixation is based on the maximum stability with the minimal hardware to be used. This is called functionally stable fixation [27]. There is no standardization for the strategic fixation of zygomatico-maxillary complex fractures. Gahari et al., 2019 [28] 16 investigated eight articles, five out of eight revealed that 3 point fixation was superior to 2 point fixation for the treatment of zygomatico-maxillary complex fractures. In our study 14 patient needed 3-point fixation, while 11 patients needed 4-point fixation with $56 \%$ and $44 \%$ respectively.

The evidence of using the advanced 3D technologies is still under great concern. Many factors are considered a challenge: Criteria of using the $3 \mathrm{D}$ technologies, the relation between local in house printing and outsourcing to industry, the time needed for the production and its delivery, its value in medical education, the optimal biomaterials to be used for 3DPCIs, and short and long term results [29,7-10,16,30].

We printed the 3 dimension models using local private companies, it took 24 to 48 hours from processing the DICOM files. We used the 3 dimensional models as a scaffold to pre bend the plate and screws and the titanium mesh used according to the reconstructed site. 16 titanium mesh placements were used in all patients, 14 orbital, 7 forehead and 6 for maxillary reconstruction. 3 patients used all the 3 types of meshes, 3 patients used orbital and forehead meshes only. 1 patient needed orbital and maxillary mesh and 8 patients needed no meshes at all. There was no recorded case with infection or extrusion which is the most common complication recorded in literature.

There are inherent drawbacks to the use of advanced 3D computer technology, including potentially increased cost, the risk of infection or extrusion of alloplastic biomaterials, and unexpected discrepancies between simulated and actual operative results $[\mathbf{7 - 1 0 , 1 3 , 1 9 ]}$. Employing 3D tech- 
nologies also does not absolve the surgeon from the responsibility of sound clinical judgment, planning, and execution. The educational role of 3D technology in craniofacial reconstruction also continues to be defined. Soft tissue atrophy is of utmost importance to be considered in the preoperative surgical planning, we recorded two patient with frontal and periorbital soft tissue atrophy which entails reconstruction with fat injection.

Previous authors support Polyether Ether Ketone (PEEK) as well suited to craniofacial reconstruction, given it approximates the physical properties of human cortical bone. Although long-term studies are still needed, this literature reports relatively low complication rates associated with PEEK implants $[\mathbf{3 1}, \mathbf{3 2}, \mathbf{3 3}, \mathbf{3 4}]$. We have not experienced complications attributed to the 3DPCI and using titanium mesh as regard implant infections or extrusions, however the PEEk is considered the best material used now, for its least complication and smooth contour.

Complex zygomatico-maxillary fractures are a common finding. The prevalence has been reported to reach up to $40 \%$ of facial fractures. Various nomenclatures have been applied to coin the fractures of this region. It has been the consensus of many authorities that the term ZMO (zygomatico maxillary orbital complex) is the most accurate term. This is because it delineates the difference between the isolated simple zygomatic arch fractures and the more complex forms of ZMO [35].

Fractures in this region are associated with changes in facial appearance complications such as hypoglobus, diplopia or changes in facial geometry might occur [36,37]. The complex anatomy of the zygoma contributed to the formation of the malar eminence. Furthermore, it participates in the formation of a part of the orbital cavity. Hence, trauma to this bony complex results in derangement in both form and function. Reestablishing the form and anatomical position of this traumatized bone is a paramount to a successful repair [38].

The primary outcome in surgical treatment of ZMO fractures focuses on restoration of facial symmetry $[39,12]$. Differences within the range of $2 \mathrm{~mm}$ in facial symmetry are virtually imperceptible and the face is considered symmetric [40]. The current study found that the There was no statistically significant difference between healthy and fixed orbits volumes. The overall deviation mean from normal was calculated to be $1.41 \pm 0.15 \mathrm{~mm}$. which lies within the acceptable range as it will not result in a virtually perceptible deformity.
Applying the principles of 3 dimention preformed meshes and pre bent plates has become the standard practice in many centers. It has been documented that high resolution preoperative 3 dimension imaging followed by 3 dimesion planning can minimize the advent of asymmetrical outcomes $[41,42]$. The use of pre-fabricated hardware and surgical navigation techniques have become an integral adjunct in complicated cases $[\mathbf{4 3}, \mathbf{4 4 , 4 5}$. Studies have documented that the application of these techniques minimize the deviation from the facial angles and orbital volume of the unaffected side [46].

The methodology utilized in this study is in accordance with many previous work groups who attempted comparative assessment of the repaired side to the unaffected side [47]. The mean of the calculated orbital volume closely resembles that calculated in Nada et al., study (The mean (SD) orbital volume was $27.9(4.0) \mathrm{cm}^{3}$ before operation and $27.5 \mathrm{~cm}^{3}(4.1)$ postoperatively $(t=0.959 ; p=$ 0.338 ). However other studies have showed different measurements. Olivera et al., 2019 investigated twenty-four orbits. The Mean orbital volume (SD) was $24.02(2.43) \mathrm{cm}^{3}$. Despite attempting to account for inter observer error the study proved that the measurements were highly reproducible with minimum error [10]. In our study we found the volume of the orbit to be $33174.14 \pm 3508.97 \mathrm{~mm}^{3}$ $(p$-value $=0.580)$. Calculation of the orbit can be highly variable as it is based on obliterating the foramina of the orbit on the software.

The calculation of orbital volume as a reference to accurate orbital repair is not without scrutiny. Olivera et al., concluded in 2019 that "Although reproducible and reliable, radiological volume assessments have not yet shown a clear correlation with clinical outcomes and postoperative management decisions should be based mainly on clinical findings [10].

\section{Conclusion and recommendation:}

The advocation of the virtual planning techniques, three dimension printing and printed custom implant enabled an accurate reduction and fixation procedure of complex acute and chronic complex upper and midface fracture, which is reflected in very satisfactory aesthetic outcome. The deviation from normal was imperceptible to the observer which is one of the primary outcomes in these surgical procedures. Calculation of the orbital volume does not have a clinical reflection although it is necessary to enable the calculations of the deviation from normal unaffected side. The three 
dimension model printing doesn't consider the soft tissue injury which is difficult to be anticipate, however three dimension reconstruction could be investigated in further studies to improve the surgical outcome. The use of at home three dimensional printing is very useful as regard time and cost management, which should be further investigated for the possibility of its introduction. Despite the high cost of printing the PEEK material we need to investigate its cost efficacy in further studies.

\section{REFERENCES}

1- Wang L.Y., Du H.M., Zhang G., Tang W., Liu L., Jing W. and Long J.: The application of digital surgical diagnosis and treatment technology:a promising strategy for surgical reconstruction of craniomaxillofacial defect and deformity, Med. Hypotheses, Vol. 77, pp. 1004-1005, 2011.

2- Drake V.E., Rizzi C.J., Greywoode J.D., Vakharia K.T. and Vakharia K.T.: Midface Fracture Simulation and Repair:A Computer-Based Algorithm. Craniomaxillofac Trauma Reconstr., Vol. 12, pp. 14-19, 2019.

3- Day K.M., Gabrick K.S. and Sargent L.A.: Applications of Computer Technology in Complex Craniofacial Reconstruction. Plast. Reconstr. Surg. Glob Open, Vol. 6: p. e1655, 2018.

4- Day K.M., Phillips P.M. and Sargent L.A.: Correction of a Posttraumatic Orbital Deformity Using ThreeDimensional Modeling, Virtual Surgical Planning with Computer-Assisted Designand Three-Dimensional Printing of Custom Implants, Craniomaxillofac. Trauma Reconstr., Vol. 11: pp. 78-82, 2018.

5- Volpe Y., Furferi R., Governi L., Uccheddu F., Carfagni M., Mussa F., Scagnet M. and Genitori L.: Surgery of complex craniofacial defects: A single-step AM-based methodology. Comput Methods Programs Biomed., Vol. 165: pp. 225-233, 2018.

6- Park S.W., Choi J.W., Koh K.S. and Oh T.S.: MirrorImaged Rapid Prototype Skull Model and Pre-Molded Synthetic Scaffold to Achieve Optimal Orbital Cavity Reconstruction. J. Oral Maxillofac. Surg., Vol. 73: pp. 1540-1553, 2015.

7- Bauermeister A.J., Zuriarrain A. and Newman M.I.: Threedimensional printing in plastic and reconstructive surgery: A systematic review. Ann. Plast. Surg., 2015.

8- Kamali P., Dean D., Skoracki R., et al.: The current role of threedimensional printing in plastic surgery. Plast. Reconstr. Surg., 137: 1045-1055, 2016.

9- Gerstle T.L., Ibrahim A.M., Kim P.S., et al.: A plastic surgery application in evolution: Three-dimensional printing. Plast. Reconstr. Surg., 133: 446-451, 2014.

10- Pfaff M.J. and Steinbacher D.M.: Plastic surgery applications using Three-dimensional planning and computerassisted design and manufacturing. Plast. Reconstr. Surg., 137: 603e-616e, 2016.

11- Darwood A., Collier J., Joshi N., et al.: Re-thinking 3D printing: A Novel approach to guide facial contouring. J. Craniomaxillofac. Surg., 43: 1256-1260, 2105.
12- Farré-Guasch E., Wolff J., Helder M.N., et al.: Application of additive manufacturing in oral and maxillofacial surgery. J. Oral Maxillofac. Surg., 73: 2408-2418, 2015.

13- Fisher M., Medina M. $3^{\text {rd }}$, Bojovic B., et al.: Indications for computer- aided design and manufacturing in congenital craniofacial reconstruction. Craniomaxillofac. Trauma Reconstr., 9: 235-241, 2016.

14 - Hyung W.Y., Nguyen A. and Yong K.K.: Facial contouring surgery with custom silicone implants based on a 3D prototype model and CT-scan: A preliminary study. Aesth. Plast. Surg., 39: 418-424, 2105.

15- Joffe J., Harris M., Kahugu F., et al.: A prospective study of computer-aided design and manufacture of titanium plate for cranioplasty and its clinical outcome. Br. J. Neurosurg., 13: 576-580, 1999.

16- Levine J.P., Patel A., Saadeh P.B., et al.: Computer-aided design and manufacturing in craniomaxillofacial surgery: the new state of the art. J. Craniofac. Surg., 23: 288-293, 2012.

17 - Müller A., Krishnan K.G., Uhl E., et al.: The application of rapid prototyping techniques in cranial reconstruction and preoperative planning in neurosurgery. J. Craniofac. Surg., 14: 899-914, 2003.

18- Poukens J., Haex J. and Riediger D.: The use of rapid prototyping in the preoperative planning of distraction osteogenesis of the cranio-maxillofacial skeleton. Comput Aided Surg., 8: 146-154, 2003.

19- Rustemeyer J., Melenberg A. and Sari-Rieger A.: Costs incurred by applying computer-aided design/computeraided manufacturing techniques for the reconstruction of maxillofacial defects. J. Craniomaxillofac. Surg., 42: 2049-2055, 2014.

20- Rotaru H., Băciut G., Stan H., et al.: Abstract: Reconstruction of craniofacial bone defects with threedimensional custommade implants. A five year experience. Intl. J. Oral Maxillof. Surg., 38: 500, 2009.

21- Tang W., Guo L., Long J., et al.: Individual design and rapid prototyping in reconstruction of orbital wall defects. J. Oral Maxillofac. Surg., 68: 562-570, 2010.

22- Tepper O.M., Sorice S., Hershman G.N., et al.: Use of virtual 3-dimensional surgery in post-traumatic craniomaxillofacial reconstruction. J. Oral Maxillofac. Surg., 69: 733-741, 2011.

23- Tsuji M., Noguchi N., Ihara K., et al.: Fabrication of a maxillofacial prosthesis using a computer-aided design and manufacturing system. J. Prosthodont., 13: 179-183, 2004.

24- Yan A. and Yaremchuk M.: Abstract P60: Facial skeletal augmentation with custom implants using computerassisted design (CAD) and computer-assisted manufacturing (CAM) technology. Plast. Recon. Surg., 135: 1239, 2015.

25- Zopf D.A., Mitsak A.G., Flanagan C.L., et al.: Computer aided-designed, 3-dimensionally printed porous tissue bioscaffolds for craniofacial soft tissue reconstruction. Otolaryngol. Head Neck Surg., 152: 57-62, 2015.

26- Bradley Strong, et al.: Bradley Strong E and Gary C. Facial Plast. Surg. Clin. N. Am., 25: 547-562, 2017. 
27- Gadkari N., Bawane S., Chopra R., Bhate K. and Kulkarni D.: Comparative evaluation of 2-point vs 3-point fixation in the treatment of zygomaticomaxillary complex fractures - A systematic review. J. Craniomaxillofac. Surg., 47 (10): 1542-1550, 2019.

28- Parthasarathy J.: 3D modeling, custom implants and its future perspectives in craniofacial surgery. Ann. Maxillofac. Surg., 4: 9-18, 2014.

29- Ozbolat I.T. and Yu Y.: Bioprinting toward organ fabrication: Challenges and future trends. IEEE Trans Biomed Eng., 60: 691-699, 2013.

30- Tan K.H., Chua C.K., Leong K.F., et al.: Scaffold development using selective laser sintering of polyetheretherketone-hydroxyapatite biocomposite blends. Biomaterials, 24: 3115-3123, 2003.

31- Alonso-Rodriguez E., Cebrián J.L., Nieto M.J., et al.: Polyetheretherketone custom-made implants for craniofacial defects: Report of 14 cases and review of the literature. J. Craniomaxillofac. Surg., 43: 1232-1238, 2015.

32- Guevara-Rojas G., Figl M., Schicho K., et al.: Patientspecific polyetheretherketone facial implants in a computer-aided planning workflow. J. Oral Maxillofac. Surg., 72: 1801-1812, 2014.

33- Rammos C.K., Cayci C., Castro-Garcia J.A., et al.: Patientspecific polyetheretherketone implants for repair of craniofacial defects. J. Craniofac. Surg., 26: 631-633, 2015.

34- Gellrich N.C., Schramm A., Hammer B., et al.: Computerassisted secondary reconstruction of unilateral posttraumatic orbital deformity. Plast. Reconstr. Surg., Vol. 110: pp. 1417-1429, 2002.

35- Rana M., Gellrich M.M. and Gellrich N.C.: Customised reconstruction ofthe orbital wall and engineering of selective laser melting (SLM) coreimplants., Br. J. Oral Maxillofac. Surg., 53, Vol. 53: pp. 208-209, 2015.

36- Farber S.J., Nguyen D.C., Skolnick G.B., Woo A.S. and Patel K.B.: Current Management of Zygomaticomaxillary Complex Fractures: A Multidisciplinary Survey and Literature Review. Craniomaxillofac. Trauma Reconstr., Vol. 9: pp. 313-322, 2016.

37- Xi Gong, Yang He, Jingang An, Yao Yang, Xiuling Huang, Meng Liu, Yangyang Zhao and Yi Zhang: Application of a Computer-Assisted Navigation System (CANS) in the Delayed Treatment of Zygomatic Fractures: A Randomized Controlled Trial. J. Oral Maxillofac. Surg., Vol. 75: pp. 1450-1463, 2017.

38- Ellis E. and KittidumkerngW.: Analysis of treatment for isolated zygomaticomaxillary. J. Oral Maxillofac. Surg., Vol. 54: pp. 386-391, 1996.

39- Kozakiewicz M. and Szymor P.: Comparison of pre-bent titanium mesh versus polyethylene implants in patient specific orbital reconstructions. Head Face Med., Vol. 9: p. 32, 2013.

40- Huempfner-Hierl H., Doerfler H.M., Kruber D., et al.: Morphologic comparison of preformed orbital meshes. J. Oral Maxillofac. Surg., Vol. 73: pp. 1119-1123, 2015.

41- Chi M.J., Ku M., Shin K.H., et al.: An analysis of 733 surgically treatedblowout fractures, Ophthalmologica, 167-175, Vol. 224, 2010.

42- Rana M., Essig H., Rüecker M., et al.: Development and demonstration of anovel computer planning solution for predefined correction of enophthalmos in anophthalmic patients using prebended 3D titanium-meshes-atechnical note. J. Oral Maxillofac. Surg., Vol. 70: pp. 631-638, 2012.

43- Zizelmann C., Gellrich N.C., Metzger M.C., et al.: Computer-assisted recon-struction of orbital floor based on cone beam tomography, Br. J. Oral Maxillofac. Surg., Vol. 45: pp. 79-80, 2007.

44- Rana M., Chui C.H., Wagner M., et al.: Increasing the accuracy of orbitalreconstruction with selective lasermelted patient-specific implantscombined with intraoperative navigation. J. Oral Maxillofac. Surg., Vol. 73: pp. 1113-1118, 2015.

45- Ranaa M., Holtmann H., Rana M., Kanatas A.N., Singh, Sproll C.K., Kübler N.R., Ipaktchi R., Hufendiek K. and Gellrich N.-C.: Primary orbital reconstruction with selective laser melted core patient-specific implants: Overview of 100 patients., Br. J. Oral Maxillofac. Surg., 2019.

46- Gomes de Oliveira P., Perry da Câmara C. and Valejo Coelho P.: Intra- and interreader variability of orbital volume quantification using 3D computed tomography for reconstructed orbital fractures. J. Craniomaxillofac. Surg., Vol. 47: pp. 1060-1064, 2019. 